\title{
Experience Feedback Committee: a management tool to improve patient safety in mental health
}

\author{
Bastien Boussat ${ }^{1,2^{*}}$, Thierry Bougerol ${ }^{3 \dagger}$, Olivier Detante ${ }^{4 \dagger}$, Arnaud Seigneurin ${ }^{1,2 \dagger}$ and Patrice François ${ }^{1,2+}$
}

\begin{abstract}
Background: A management tool, called the Experience Feedback Committee, has been applied for patient safety and successfully used in medical departments. The purpose of this study was to analyse the functioning of an Experience Feedback Committee in a psychiatric department and to explore its contribution to the particular issues of patient safety in mental health.
\end{abstract}

Methods: We conducted a descriptive study based on all the written documents produced by the Experience Feedback Committee between March 2010 and January 2013. The study was conducted in Grenoble University Hospital in France. We analysed all reported incidents, reports of meetings and event analysis reports. Adverse events were classified according to the Conceptual Framework for the International Classification for Patient Safety.

Results: A total of 30 meetings were attended by 22 professionals including seven physicians and 12 paramedical practitioners. We identified 475 incidents reported to the Experience Feedback Committee. Most of them (92\%) had no medical consequence for the patient. Eleven incidents were investigated with an analysis method inspired by civil aviation security systems. Twenty-one corrective actions were set up, including eight responses to the specific problems of a mental health unit, such as training to respond to situations of violence or management of suicide attempts.

Conclusions: The Experience Feedback Committee makes it possible to involve mental healthcare professionals directly in safety management. This tool seems appropriate to manage specific patient safety issues in mental health.

Keywords: Risk management, Mental health, Neuropsychiatry, Quality improvement, Interdisciplinary communication

\section{Background}

Patient safety has become a public health priority in the past 15 years since the publication of major epidemiological studies on healthcare-related adverse events [1-5]. The last Canadian national study estimated that 7.5 adverse events occurred for 100 hospital admissions, including a high proportion of preventable events and adverse events leading to death [6].

Even if many adverse events are similar in all medical units, there are specific patient safety issues in mental

\footnotetext{
*Correspondence: BBoussat@chu-grenoble.fr

'Thierry Bougerol, Olivier Detante, Arnaud Seigneurin and Patrice François contributed equally to this work

${ }^{2}$ Laboratory TIMC, UMR 5525, CNRS, Joseph Fourier University, Grenoble, France

Full list of author information is available at the end of the article
}

health. Indeed, neuropsychiatric units face events caused by behavioural problems such as violence, absconding, self-harm and suicide attempts [7-11]. Mental health adverse events result from multiple factors, mixing human behaviour risks and healthcare organizational weaknesses. Considering the lack of readily available information to guide patient safety systems in mental health, the improvement of management tools is essential to promote a patient safety culture among healthcare professionals [11-13].

Since the 1970s, civil aviation has developed operating experience feedback to improve passenger safety. Air transport safety systems require that any incident, even minor, must be treated by a systemic analysis within the air crew. Inspired by those security systems, 
a specific mechanism, called the Experience Feedback Committee (EFC), was created in 2005 to analyse adverse events within a medical team. In France, the method was adapted to healthcare facilities with the help of Air France Consulting and was successfully implemented in an emergency department and a radiotherapy unit [14-19]. The EFC is a team composed of professionals representing the diversity of the functions encountered in the medical unit. The EFC members meet monthly to examine adverse events reported to their medical unit. An event requiring a thorough analysis is chosen at each meeting according to its severity and frequency and corrective actions are suggested based on the results of the analysis. The main principles of the method are managing patient safety within a medical team and setting up corrective actions concerning latent factors that contributed to the occurrence of events or near-miss events.

The aim of this study was to describe the functioning of the EFC in a neuropsychiatric department and to discuss its contribution to the management of patient safety.

\section{Methods}

Study design

We conducted a descriptive study based on the written reports of the neuropsychiatric department EFC from its beginning in March 2010 until January 2013.

\section{Setting}

The study was conducted in a 1347-bed acute-care university hospital. The neuropsychiatry department has an annual patient volume of 4800 stays.

Adverse events and near-misses affecting patients (such as nosocomial infections, technical complications, negligence, diagnostic mishaps, therapeutic incidents, etc.) are reported by healthcare professionals, through a voluntary internal reporting system, to the hospital's central safety unit using a standardized report form. This unit is composed of a medical doctor, a pharmacist and an engineer specializing in quality management. The reports of events classified by severity and risk areas are presented during a weekly meeting involving representatives of the administration and professionals in charge of specific risk areas such as the risks associated with drugs (pharmacovigilance), nosocomial infections (infection vigilance), healthcare materials and devices (medical device vigilance), transfusion (haemovigilance), etc. The central safety unit directly investigates the most serious events and those involving several hospital departments. Other events are transmitted to the appropriate operator and to executives of relevant departments. For departments where an EFC has been implemented, the central safety unit addresses the reports of events to the EFC leader every month.

\section{Neuropsychiatric department EFC}

The neuropsychiatric department EFC was set up in March 2010 and works through a written procedure in accordance with the method proposed by Air France Consulting [17, 18, 20]. The Committee is composed of volunteer representatives of the various professions within the neuropsychiatric division. A few days before the committee meeting, the EFC leader receives a file with event reports concerning the neuropsychiatric division. Committee meetings are conducted according to a standardized framework: (1) reading the list of reported events, (2) choosing a priority event to investigate by consensus according to the criticality of each incident, (3) choosing the professional responsible for the investigation, (4) reviewing the analysis made of the event chosen the previous month, (5) choosing corrective actions and (6) monitoring on-going actions. The investigation is carried out during the month following the EFC by a designated person using a method, called ORION@, developed from methods of systemic analysis used in civil aviation and adapted to the healthcare domain by Air France Consulting [14-17]. Previously trained investigators must follow the main steps of the ORION৫ method to fill out a standardized report (Additional file 1: Appendix): collecting data and existing recommendations, describing the chronological facts that occurred before, during and after the event, describing the failures, looking for causes of errors and latent factors that could have contributed to the failures, setting up corrective actions and writing a report of the analysis. Causes and latent factors are sought in different areas such as political, organizational, working conditions, team functioning, procedures, actors and the patient.

\section{Data collection}

All written documents from the EFC of the neuropsychiatric division were analysed. The events reported were classified according to the source of the report, the type of event and the consequence for the patient using the International Classification for Patient Safety [21]. Written reports from meetings were analysed using a standardized form that included the theoretical steps of an EFC meeting and the contents of the ORION@ analysis (as described above). All documents were analysed by two independent investigators. Differences in rating were discussed until a consensus was reached.

\section{Statistical analysis}

We reported the characteristics of the EFC's main functioning (meetings and participants), the adverse events reported and the analysis reports as medians and interquartile ranges (IQR; i.e., 25th and 75th percentiles) for continuous variables and number and percentages for 
categorical variables. The analysis was performed using $\mathrm{R}$ version 3.0.1.

\section{Results}

The committee set up 30 meetings during the study period. A total of 22 professionals participated in the EFC (Table 1), including seven physicians, four head nurses, four auxiliary nurses, three nurses, one secretary, one physical therapist, one cleaning staff member and one quality engineer. The median number of attendants was eight (IQR 6-9) per meeting. A report was written for each meeting. Priority events were chosen in half of the meetings, analysis reports were presented and corrective actions were decided in more than one-third of the meetings (Table 1 ). The previous corrective actions were monitored in 19 meetings (63.3\%).

A total of 475 reported incidents were transmitted to the EFC (Table 2). A median number of 12 incidents (IQR 7-20) were discussed per meeting. Incidents were mainly $(97.1 \%)$ reported by a professional of the department and $93.3 \%$ of them occurred inside the department. Reported incidents concerned mainly clinical administration (29.3\%) (including incidents in patient identification, patient transfer, admission, discharge), behaviour (24\%) (concerning patient or staff) and patient accidents (12\%). The majority of incidents had no clinical consequence for the patient $(91.8 \%)$ or the care process (70.1\%). In 29 cases (6.1\%), the reported event had a mild or moderate consequence for the patient (Table 2). Among the 20 events involving a mild consequence for the patient, ten events were related to a patient fall, four events concerned violence against the staff or another patient, three events were related to inadequate equipment and three events were suicide attempts. Among the nine events involving a moderate consequence, eight falls led to orthopaedic fracture or head trauma, and one admission error concerned a patient hospitalized in a corridor.

Fifteen priority incidents were chosen for investigation, including six incidents related to clinical administration, four incidents related to behaviour problems, four incidents related to infrastructure and fixtures and one to the keeping of archives. Four incidents chosen were not investigated. Among the 11 analyses carried out, nine reports were written, whereas two reports were only oral (Table 3). Three reports involved a problem of coordination with other hospital departments. First, an error of emergency transfer was reported for a patient hospitalized in a corridor who was transported to the intensive care unit for optimal surveillance because of loss of consciousness. Second, stretchers for an imaging examination emergency were recurrently unavailable. Third, organizational problems of the psychiatric consultation were reported. Three reports analysed adverse events associated with behavioural disorders: the inability of a professional to properly manage a patient with a suicide

Table 1 Main functioning characteristics of the Experience Feedback Committee of the neuropsychiatry department

\begin{tabular}{|c|c|c|}
\hline & $N=22$ & $\%$ \\
\hline \multicolumn{3}{|l|}{ Participants } \\
\hline Physicians & 7 & 31.8 \\
\hline Head nurses & 4 & 18.2 \\
\hline Auxiliary nurses & 4 & 18.2 \\
\hline Nurses & 3 & 13.6 \\
\hline Secretary & 1 & 4.5 \\
\hline Quality engineer & 1 & 4.5 \\
\hline Physical therapist & 1 & 4.5 \\
\hline Cleaning staff member & 1 & 4.5 \\
\hline Median number of participations per participant (IQR 25-75) & 9 & $(5-15)$ \\
\hline \multirow[t]{2}{*}{ Median number of participants per meeting (IQR 25-75) } & 8 & $(6-9)$ \\
\hline & $N=30$ & $\%$ \\
\hline \multicolumn{3}{|l|}{ Meetings } \\
\hline Writing of minutes & 30 & 100.0 \\
\hline Listening to the events reported during the previous month & 30 & 100.0 \\
\hline Choosing a priority event to analyse during the following month & 15 & 50.0 \\
\hline Listening to the analysis report from the event chosen the previous month & 11 & 36.7 \\
\hline Deciding corrective actions & 11 & 36.7 \\
\hline Following up the previous corrective actions & 19 & 63.3 \\
\hline
\end{tabular}


Table 2 Characteristics of the events reported during the Experience Feedback Committee meetings

\begin{tabular}{|c|c|c|}
\hline Characteristics & $N=475$ & $\%$ \\
\hline \multicolumn{3}{|l|}{ Incident type } \\
\hline Clinical administration & 139 & 29.3 \\
\hline Behaviour & 114 & 24.0 \\
\hline Patient accidents & 57 & 12.0 \\
\hline Infrastructure/building/fixtures & 41 & 8.6 \\
\hline Medical device/equipment & 30 & 6.3 \\
\hline Resources/organizational management & 30 & 6.3 \\
\hline Nutrition & 20 & 4.2 \\
\hline Clinical process/procedure & 16 & 3.4 \\
\hline Medication/IV fluids & 14 & 2.9 \\
\hline Documentation & 11 & 2.3 \\
\hline Healthcare-associated infection & 3 & 0.6 \\
\hline Blood/blood products & 0 & 0.0 \\
\hline Oxygen/gas/vapour & 0 & 0.0 \\
\hline \multicolumn{3}{|l|}{ Degree of Harm } \\
\hline None, without care modification & 343 & 72.2 \\
\hline None, with care modification & 103 & 21.7 \\
\hline Mild & 20 & 4.2 \\
\hline Moderate & 9 & 1.9 \\
\hline Severe & 0 & 0.0 \\
\hline Death & 0 & 0.0 \\
\hline \multicolumn{3}{|l|}{ Report provider } \\
\hline Staff from the neuropsychiatric department & 461 & 97.1 \\
\hline Staff from another department & 16 & 3.4 \\
\hline \multicolumn{3}{|l|}{ Place of the event } \\
\hline In the neuropsychiatric department & 443 & 93.3 \\
\hline In another department & 32 & 6.7 \\
\hline
\end{tabular}

risk in child psychiatry, an attempted suicide by strangulation with a phone cord and finally the investigation of repeated fugues in adult psychiatry. Three ORION@ analyses were carried out to investigate a technical equipment failure including an ECG machine, the computer network and a power failure. The last reports concerned a loss of patient records, a delivery mistake for special meals and the fall of an elderly patient.

The expertise of the written reports showed that the ORION@ method was frequently followed. The chronology of the facts and the identification of contributing or latent factors were described in $80 \%$ of the cases. However, existing recommendations were only described in one-half of the cases. Twenty-six corrective actions were proposed by the professionals who performed the analyses. The committee decided to implement 21 actions. Written guidelines $(n=11)$ were the most common type of action (see box). Other actions included staff training, improvement of the availability of material resources and a deeper analysis of an event in the context of a medical thesis. For example, the ORION॰ report related to the fall of an elderly patient showed several factors contributing to the event: some factors related to the patient (inappropriate behaviour of a patient with a depressive syndrome, decreased alertness and reflexes due to anxiolytic treatment) and organizational factors (lack of assessment of the risk for falls at admission, lack of supervision due to a high level of department activity). The corrective actions selected were structured around these two main types of factors. A systematic screening of risk factors for falling at admission was established. Secondary prevention actions were also decided: pharmaceutical adaptation to reduce iatrogenic events and implementation of monitoring for high-risk patients by a team composed of a physiotherapist, an occupational therapist and a movement therapist. Finally, adjustments were made at the facility level with adjustable-height beds, night lights in the rooms and more convenient showers.

\section{Box Corrective actions set up}

Guideline writing
Inpatient transfer from ED
Patient medical record management
Suicide risk assessment at admission
Management of patient with behaviour problems
Security guards system
Job profile of secretary
Failing risk assessment at admission
Protocol to prevent failing risk
User manual for ECG advise
Emergency consultation procedure
Electrical failure procedure
Training
Respond to situations of violence
Management of elderly patients
Respond to suicide attempt
Proper management of medical records
Material resources
Establishment of an isolation room
Change of ECG device
Loan of two ECG device
Implementation of a planning software for inpatient transport
Research
Completion of a medical thesis on inpatient transfers in the hospital

\section{Discussion}

This study highlighted that the EFC implemented in the mental health department functions routinely with patient safety incidents analysed and corrective actions set up. The EFC method, which was successfully implemented in medical units, is also relevant to managing patient safety in mental health. Nath and Marcus 
Table 3 Characteristics of the analysis reports and of the corrective actions

\begin{tabular}{|c|c|c|}
\hline & $N=11$ & $\%$ \\
\hline \multicolumn{3}{|l|}{ Analysis reports } \\
\hline Written reports & 9 & 81.8 \\
\hline Oral reports & 2 & 18.2 \\
\hline Description of the data collection method & 9 & 81.8 \\
\hline Individual interviews & 9 & 81.8 \\
\hline Collective debriefing & 1 & 9.1 \\
\hline Files & 5 & 45.5 \\
\hline Area visits & 6 & 54.5 \\
\hline Description of the chronology of facts & 9 & 81.8 \\
\hline Description of existing recommendations & 6 & 54.5 \\
\hline Error identification & 6 & 54.5 \\
\hline Identification of contributing or latent factors & 10 & 90.9 \\
\hline Management & 5 & 45.5 \\
\hline Organization and procedures & 7 & 63.6 \\
\hline Working environment & 6 & 54.5 \\
\hline Teamwork & 3 & 27.3 \\
\hline Technical processes & 5 & 45.5 \\
\hline Professionals & 4 & 36.4 \\
\hline Patients & 2 & 18.2 \\
\hline \multicolumn{3}{|l|}{ Corrective actions } \\
\hline Proposed actions & $N=26$ & \\
\hline Staff Training & 4 & 15.4 \\
\hline Writing procedures & 12 & 46.1 \\
\hline Organizational changes & 4 & 15.4 \\
\hline Increasing material resources & 5 & 19.2 \\
\hline Decided actions & $N=21$ & \\
\hline With a professional in charge & 14 & 66.7 \\
\hline From the department & 12 & 57.1 \\
\hline From another department & 2 & 9.5 \\
\hline With a defined deadline & 9 & 42.9 \\
\hline
\end{tabular}

demonstrated that some patient safety incidents and contributing factors are specific to mental health [11]. Although the EFC examined events that might have been found in other medical units, events specific to mental health were also reported and analysed. For example, suicide attempts and missing person incidents were investigated and the analyses highlighted several organizational flaws in suicide risk assessment, monitoring patients at risk and securing the department's premises. Consequently, actions concerning the security of the building and the assessment of suicide risk in child psychiatry (specific staff training to manage patients with violent behaviour and improved guidelines) were set up.

Considering patient safety incidents in mental health as a result of a complex set of contributing factors, the EFC provided a structured framework to analyse them within the department's routine $[8,13,22,23]$. The principle is to choose only one event per meeting to perform a thorough analysis using the ORION@ method. As advocated by the Reason model, this method aims to identify factors related to the design of the system's organization or the workplace environment rather than individual error [24]. The method implies the main steps of the Association of Litigation and Risk Management (ALARM) protocol but seems easier to use for healthcare professionals who are not specialists in risk management [25]. The essential contribution of an EFC is to provide a formal framework to correct the latent failures in the department's organization.

Over the 3 years studied, the theoretical framework for conducting an EFC was not always followed. Indeed, the analysis of events did not always include all the steps defined in the ORIONৎ method and the search for contributing factors was often superficial. These deviations can be partly explained by the staff's lack of time and availability. Carrying out the investigations to determine the causes of events as well as writing the report is timeconsuming. Inconsistent monitoring of the corrective actions previously set up resulted in the resurgence of certain patient safety incidents that had previously been investigated, highlighting the importance of monitoring corrective actions by the EFC. A low level of expertise in conducting the analyses may also explain these deviations: only professionals involved in the EFC at its beginning were given formal training, and additional training was not proposed afterwards. Consequently, regular training seems necessary to ensure the quality of meetings and event analysis.

To function properly, the EFC requires reports of adverse events experienced by professionals. Several studies have shown that healthcare professionals, particularly physicians, agree with the importance of incident reporting and the concept of learning from errors $[26,27]$. Nevertheless, in practice, many incidents are not reported [28, 29]. Self-report of patient safety incidents is hindered by several barriers such as time constraints, complex forms, fear of punishment, shame as well as lack of education and feedback [28, 30-33]. In the present study, we were not able to estimate the proportion of unreported incidents. However, the committee had enough incidents to discuss every month. Mental healthcare professionals are probably informed more easily of the corrective actions set up and can observe their effects considering that reported incidents are analysed by professionals in the department. Consequently, the existence of an EFC in a mental health department may improve incident reporting.

Psychiatric units provide global and coordinated care for patients through the involvement of many professionals. However, Priest and Borella [34] showed a higher risk of incidents due to the increasing distribution of patient 
care over multiple practitioners. One of the strengths of the EFC is to gather all categories of professionals working in the unit and reinforcing interprofessional collaboration and promoting teamwork. This multidisciplinary approach also contributes to identifying system vulnerabilities more easily [35].

This study had several limitations. First, the functioning of an EFC depends on the professionals involved and the study was conducted in only one department. Second, the reporting system based on self-reporting by healthcare professionals did not provide the proportion of unreported incidents and did not take into account the patient's complaints that were treated by the hospital's legal department. Consequently, we were not able to measure the impact of the EFC on the completeness of incident reports and more generally on the prevalence of adverse events. Third, the impact of the EFC on patient safety was not assessed using clinical outcomes. However, we assumed that corrective actions against identified vulnerabilities resulted in an improvement of patient safety, including an improvement in the patient safety culture among professionals attending the committee.

\section{Conclusions}

The EFC is a tool allowing the direct involvement of mental health professionals to manage patient safety. This innovative management tool is adapted to the specific adverse events encountered in mental health. The theoretical framework for conducting an EFC was not always followed, suggesting the need for simplifying the method for professionals subject to tight time constraints.

\section{Additional file}

Additional file 1: Appendix. ORION standardized report

\begin{abstract}
Author's contributions
BB: data management, analysis and interpretation; manuscript drafting; final approval of the version to be published; agreement to be accountable for all aspects of the work. TB: contribution to the design of the work; revising for important intellectual content; final approval of the version to be published; agreement to be accountable for all aspects of the work. OD: contribution to the design of the work; revising for important intellectual content; final approval of the version to be published; agreement to be accountable for all aspects of the work. AS: data analysis and interpretation; revising for important intellectual content; final approval of the version to be published; agreement to be accountable for all aspects of the work. PF: contribution to the design of the work; data analysis and interpretation; revising for important intellectual content; final approval of the version to be published; agreement to be accountable for all aspects of the work.
\end{abstract}

\section{Author details}

${ }^{1}$ Public Health Department, Grenoble University Hospital, Grenoble, France. ${ }^{2}$ Laboratory TIMC, UMR 5525, CNRS, Joseph Fourier University, Grenoble, France. ${ }^{3}$ Psychiatry Department, Grenoble University Hospital, Grenoble, France. ${ }^{4}$ Neurology Department, Grenoble University Hospital, Grenoble, France.

\section{Acknowledgements}

We acknowledge Linda Northrup for her assistance in editing the manuscript. We acknowledge all professionals who participated in the EFC: C. Besançon, M. Bonnet, I. Bouillaud, C. Boutte, D. Chergui, F. Dejoie, O. Detante, M. Dubuc, C. Girard, S. Im, F. Imburchia, E. Lagrange, J.C. Peyrin, M. Poullet, C. Rivière, C. Robart, E. Ruhaud, E. Sellier, S. Sevenier, B. Teppa, C. Vidy, G. Weil.

\section{Funding}

This work was supported by the French Ministry of Health, project PREPS 2012.

\section{Compliance with ethical guidelines}

\section{Competing interests}

The authors declare that they have no competing interests

Received: 27 January 2015 Accepted: 20 August 2015

Published online: 03 September 2015

\section{References}

1. Brennan TA, Leape LL, Laird NM, Hebert L, Localio AR, Lawthers AG et al (2004) Incidence of adverse events and negligence in hospitalized patients: results of the Harvard Medical Practice Study I. Qual Health Care 13(2):145-151. doi:10.1136/qshc.2002.003822

2. Pietro DA, Shyavitz LJ, Smith RA, Auerbach BS (2000) Detecting and reporting medical errors: why the dilemma? BMJ 320(7237):794-796

3. Reason J (2000) Human error: models and management. BMJ 320(7237):768-770

4. Vincent C, Neale G, Woloshynowych M (2001) Adverse events in British hospitals: preliminary retrospective record review. BMJ 322(7285):517-519

5. Weingart SN, Wilson RM, Gibberd RW, Harrison B (2000) Epidemiology of medical error. BMJ 320(7237):774-777

6. Baker GR, Norton PG, Flintoft V, Blais R, Brown A, Cox J et al (2004) The Canadian Adverse Events Study: the incidence of adverse events among hospital patients in Canada. CMAJ Can Med Assoc J 170(11):1678-1686

7. Bowers L, Simpson A, Alexander J (2003) Patient-staff conflict: results of a survey on acute psychiatric wards. Soc Psychiatry Psychiatr Epidemiol 38(7):402-408. doi:10.1007/s00127-003-0648-x

8. Desai RA, Dausey DJ, Rosenheck RA (2005) Mental health service delivery and suicide risk: the role of individual patient and facility factors. Am J Psychiatry 162(2):311-318. doi:10.1176/appi.ajp.162.2.311

9. Druss BG (2007) Improving medical care for persons with serious mental illness: challenges and solutions. J Clin Psychiatry 68(Suppl 4):40-44

10. Laitanantpong D (2006) The patient risk in psychiatric service at King Chulalongkorn Memorial Hospital, Thai Red Cross Society. J Med Assoc Thail Chotmaihet thangphaet 89(Suppl 3):S174-S179

11. Nath SB, Marcus SC (2006) Medical errors in psychiatry. Harv Rev Psychiatry 14(4):204-211. doi:10.1080/10673220600889272

12. Hairon N (2008) Guidelines focus on improving patient safety in mental health. Nurs Times 104(48):19-20

13. Brickell TA, McLean C (2011) Emerging issues and challenges for improving patient safety in mental health: a qualitative analysis of expert perspectives. J Patient Saf 7(1):39-44. doi:10.1097/PTS.0b013e31820cd78e

14. Woynar S, Debouck F, Cellier P, Bourhis J, Cauterman M, Lartigau E (2007) Towards global security in radiation oncology. Using event notification feed-back. Cancer Radiother J Soc Fr De Radiother Oncol 11 (6-7):320328. doi:10.1016/j.canrad.2007.09.003

15. Lartigau E, Vitoux A, Debouck F (2009) Crex and Orion analysis in radiation oncology: towards a mutualisation of corrective actions. Cancer Radiother J Soc Fr Radiother Oncol 13(6-7):458-460. doi:10.1016/j. canrad.2009.08.143

16. Debouck F, Petit HB, Lartigau E (2010) From Crex mutualisation to clinical audit. Cancer Radiother J Soc Fr Radiother Oncol 14(6-7):571-575. doi:10.1016/j.canrad.2010.07.018

17. Debouck F, Rieger E, Petit H, Noel G, Ravinet L (2012) ORION(R): a simple and effective method for systemic analysis of clinical events and precursors occurring in hospital practice. Cancer Radiother J Soc Fr Radiother Oncol 16(3):201-208. doi:10.1016/j.canrad.2011.12.002 
18. Francois P, Sellier E, Imburchia F, Mallaret MR (2013) Experience feedback committee: a method for patient safety improvement. Rev Epidemiol Sante Publique 61(2):155-161. doi:10.1016/j.respe.2012.09.001

19. Lecoanet A, Sellier E, Carpentier F, Maignan M, Seigneurin A, Francois $P$ (2013) Experience feedback committee in emergency medicine: a tool for security management. Emer Med J EMJ. doi:10.1136/ emermed-2013-202767

20. Lartigau E, Coche-Dequeant B, Dumortier V, Giscard S, Lacornerie T, Lasue A et al (2008) Experience feed back committee in radiotherapy (CREx): a compulsory tool for security management evaluation at two years. Cancer Radiother J Soc Fr Radiother Oncol 12(6-7):610-613. doi:10.1016/j. canrad.2008.07.007

21. World Alliance For Patient Safety Drafting Group, Sherman H, Castro G, Fletcher M, World Alliance for Patient Study, Hatlie M et al (2009) Towards an International Classification for Patient Safety: the conceptual framework. Int J Qual Health Care J Int Soc Qual Health Care/ISQua. 21(1):2-8. doi:10.1093/intqhc/mzn054

22. Grasso BC, Bates DW (2003) Medication errors in psychiatry: are patients being harmed? Psychiatric Serv 54(5):599

23. Measham TJ (1995) The acute management of aggressive behaviour in hospitalized children and adolescents. Can J Psychiatry Rev Can Psychiatr 40(6):330-336

24. Reason JT, Carthey J, de Leval MR (2001) Diagnosing "vulnerable system syndrome": an essential prerequisite to effective risk management. Qual Health Care QHC 10(Suppl 2):ii21-ii25

25. Vincent C, Taylor-Adams S, Chapman EJ, Hewett D, Prior S, Strange P et al (2000) How to investigate and analyse clinical incidents: clinical risk unit and association of litigation and risk management protocol. BMJ 320(7237):777-781
26. Firth-Cozens J (2003) Evaluating the culture of safety. Qual Saf Health Care 12(6):401

27. Sarvadikar A, Prescott G, Williams D (2010) Attitudes to reporting medication error among differing healthcare professionals. Eur J Clin Pharmacol 66(8):843-853. doi:10.1007/s00228-010-0838-x

28. Blendon RJ, DesRoches CM, Brodie M, Benson JM, Rosen AB, Schneider E et al (2002) Views of practicing physicians and the public on medical errors. New Engl J Med 347(24):1933-1940. doi:10.1056/NEJMsa022151

29. Haller G, Courvoisier DS, Anderson H, Myles PS (2011) Clinical factors associated with the non-utilization of an anaesthesia incident reporting system. Br J Anaesth 107(2):171-179. doi:10.1093/bja/aer148

30. Hobgood C, Weiner B, Tamayo-Sarver JH (2006) Medical error identification, disclosure, and reporting: do emergency medicine provide groups differ? Acad Emer Med Off J Soc Acad Emer Med 13(4):443-451. doi:10.1197/j.aem.2005.11.005

31. Leape LL, Berwick DM (2005) Five years after to err is human: what have we learned? JAMA 293(19):2384-2390

32. Leape LL (2002) Reporting of adverse events. New Engl J Med 347(20):1633-1638. doi:10.1056/NEJMNEJMhpr011493

33. Vincent C, Stanhope N, Crowley-Murphy M (1999) Reasons for not reporting adverse incidents: an empirical study. J Eval Clin Pract 5(1):13-21

34. Priest SL, Borella AB (1998) A crash course in systems analysis. J Healthcare Info Manag JHIM 12(3):17-23

35. Schmutz J, Manser T (2013) Do team processes really have an effect on clinical performance? A systematic literature review. Br J Anaesth 110(4):529-544. doi:10.1093/bja/aes513

\section{Submit your next manuscript to BioMed Central and take full advantage of:}

- Convenient online submission

- Thorough peer review

- No space constraints or color figure charges

- Immediate publication on acceptance

- Inclusion in PubMed, CAS, Scopus and Google Scholar

- Research which is freely available for redistribution

Submit your manuscript at

www.biomedcentral.com/submit

C BioMed Central 